\title{
A novel assay to evaluate olfactory modulation of honeybee aggression
}

\author{
Nicole M. D. van der Burg ${ }^{1}$, Nicholas Lavidis ${ }^{2}$, Charles Claudianos ${ }^{1}$, \\ Judith REINHARD ${ }^{1}$ \\ ${ }^{1}$ Queensland Brain Institute, The University of Queensland, Brisbane, QLD 4072, Australia \\ ${ }^{2}$ School of Biomedical Sciences, The University of Queensland, Brisbane, QLD 4072, Australia
}

Received 21 July 2013 - Revised 2 November 2013 - Accepted 11 December 2013

\begin{abstract}
Numerous assays exist to measure aggressive behaviour in honeybees, most of them using freeflying bees under natural conditions, with results often variable due to environmental factors. Our study describes a novel, laboratory-based Petri dish assay that uses a moving target treated with the alarm pheromone component isoamyl acetate (IAA). In this assay, aggression levels can be measured in individual bees via recording specific behaviours associated with the different stages of aggression. We used this new assay to investigate the modulating effect of the plant odours lavender and Praescent on aggression in bees, as these odours are reported to have a "calming" effect on animals. Both short-term (5 min) and long-term (48 h) exposure to lavender and Praescent attenuated aggression, even in the presence of the moving target and IAA. Plant odours may thus be an effective treatment for reducing aggressive behaviour in bees.
\end{abstract}

\section{honeybee aggression / IAA / olfaction / behaviour / plant odours}

\section{INTRODUCTION}

Aggression in honeybees is a sequence of behaviours triggered by the presence of a potential threat to the hive and initiated by the so-called guard bees, which form the first line of defence for a colony (Collins et al. 1980; Moore et al. 1987; Breed et al. 1990, 2004; Arechavaleta-Velasco and Hunt 2003; Hunt 2007). During the first stage of aggression, bees show a typical alert posture, display increased locomotion, release of alarm pheromone and wing buzzing to recruit nest mates. During the second stage when a potential target is located, bees approach it flying or running, followed by

Corresponding author: J. Reinhard, j.reinhard@uq.edu.au

Manuscript editor: Bernd Grünewald the third stage, during which bees start to display threatening behaviour towards the target, rapidly flying around it and contacting it. In the final stage, the attack culminates with bees actively biting and stinging the target.

Alerted honeybees recruit nest mates for defence of the hive using an alarm pheromone, which is produced by glands of the sting apparatus (Winston 1991). The principal active component of this pheromone is isoamyl acetate (IAA, also known as isopentyl acetate) (reviewed in Free 1987; Schmidt 1998; Breed et al. 2004; Hunt 2007). IAA has been used to represent the pheromone's effects in various aggression studies with honeybees as it triggers a similar reaction to the whole alarm pheromone (Hunt 2007). Importantly, a combination of olfactory, visual and mechanical stimuli, such as the alarm pheromone, potential smells produced by a predator, the presence of dark targets 
and, in particular, the movement of these targets is crucial to trigger the full alarm and defensive response in honeybees (reviewed in Free 1987; Schmidt 1998).

The complex nature of honeybee defensive behaviour has stimulated the development of a variety of assays to measure aggression of different bee species or different colonies, and to determine the influence of genetic and environmental factors on aggressiveness (e.g. Collins et al. 1982; Free 1987; Schmidt 1998; Guzman-Novoa et al. 2004; Breed et al. 2004). Field assays utilise free-flying bees, measuring aggression of a hive by recording the number of stings left on an object waved in front of a hive entrance or above an open hive, along with the latency time between the initial disturbance and the first attack (Free 1961; Southwick and Moritz 1987; Guzman-Novoa et al. 1999, 2003, 2004; Wager and Breed 2000). These assays are a powerful tool to decipher the sequence of events and the triggers inducing honeybee defensive behaviour (Free 1961; Wager and Breed 2000), but the results are often variable depending on a wide range of external factors, including group effects, weather conditions, seasonality and time of day (Southwick and Moritz 1985, 1987; GuzmanNovoa et al. 1999). Data on individual bees are also very difficult to obtain from such experiments, and only few assays have scored individual behaviours associated with hive defence as measure for aggressiveness (Guzman-Novoa et al. 2003). To overcome these disadvantages, laboratory-based assays with individual bees have been developed using electric shocks to trigger the stinging reflex and measure its threshold either in restrained bees (Sting-Extension-Reflex assay) or in bees walking on an electric grid in a Petri dish (Kolmes and Fergusson-Kolmes 1989; Paxton et al. 1994; Núñez et al. 1998; Balderrama et al. 2002; Lenoir et al. 2006; Uribe-Rubio et al. 2008; Roussel et al. 2009; Tedjakumala and Giurfa 2013). These assays have provided a deeper understanding about stinging response thresholds in honeybees, the genetic basis of the individual variability with regard to aggressive behaviour and the influence of the nest environment on the defensive response. However, both these assays are relying on an unnatural trigger, namely electric shock, and neither uses an object as actual target for the bees' aggression. Clearly, we still lack a controlled laboratory-based assay that reliably measures individual bees' level of aggressiveness in response to a relevant target. Such an assay would allow us to study the effect of subtle modulators of aggression, such as odours, and to investigate the neural and molecular mechanisms controlling aggressive behaviour in honeybees.

Understanding mechanisms underlying aggression in bees is of interest to scientists and beekeepers alike and is particularly relevant in areas where the highly aggressive Africanised bee (Apis mellifera scutellata) has been the cause of over one thousand human deaths and tens of thousands of livestock deaths (Breed et al. 2004; Francoy et al. 2009). An effective treatment to reduce aggression in these bees is highly sought after. Smoke is traditionally used by beekeepers around the world to reduce defensive responses in honeybees, as it decreases the response of olfactory receptor neurons to IAA (Visscher et al. 1995). However, smoke cannot be continuously applied. There are anecdotal reports of beekeepers that apply lavender oil on hands or gloves, which allegedly "calms" the bees and reduces stinging. This raises the question whether plant odours such as lavender could indeed be used to reduce aggressive behaviour in bees both in the shortand long-term. Other than lavender, there is one particular plant odour of interest, namely the odour Praescent ${ }^{\mathrm{TM}}$ (developed by N. Lavidis). This odour is a mixture of three common plant odorants (cis-3-hexanol, trans-2-hexenal and alpha-pinene), two of which are typical green leaf volatiles released when plants suffer tissue damage (Visser et al. 1979). Interestingly, Praescent and its constituents have been found to modulate anxiogenic phenotypes in rodents, significantly reducing the physiological effects 
of stress (Akutsu et al. 2002, 2003; Aou et al. 2005; Kim et al. 2005; Tokumo et al. 2006; Fukada et al. 2007; Fujita et al. 2010). With aggression being a natural behavioural response to stress, also in honeybees (Even et al. 2012), Praescent clearly is a plant odour worth investigating with respect to its potential for reducing bee aggressive behaviour.

The purpose of this study was twofold: Firstly, we developed a simple laboratorybased assay that measured aggressiveness in individual honeybees in response to a relevant target; second, using this assay, we investigated the potential modulating effect of two plant odours, lavender and Praescent, on aggressive behaviour in bees.

\section{MATERIALS AND METHODS}

\subsection{Honeybees}

Honeybees were European-derived Apis mellifera, housed in an experimental hive at the University of Queensland in Brisbane, Australia. Honeybees could freely forage on flowering plants. Experiments were conducted between March and November, during Southern hemisphere autumn, winter and spring. Due to the subtropical location of Brisbane, honeybees forage all year round in Queensland, and daily average temperatures range from 15 to $28{ }^{\circ} \mathrm{C}$.

For the initial experiments designed to develop a laboratory-based assay for measuring aggressive behaviour in honeybees (see 2.3), random bees were collected from the hive entrance using $50-\mathrm{mL}$ falcon tubes. Two bees at a time were caught and coldanaesthetised at $4{ }^{\circ} \mathrm{C}$ for approximately $10-15 \mathrm{~min}$ until completely immobilised. For the behavioural assays testing the effect of plant odours on aggressive behaviour (see 2.4 and 2.5), only guard bees were used to ensure a stable behavioural baseline. Guard bees are usually present at the hive entrance; they are the first to respond to a threat and recruit nest mates via release of the alarm pheromone (Winston 1991; Hunt 2007). Guard bees were caught by waving a black feather at the hive entrance for $5 \mathrm{~s}$. Once, about 15-30 guard bees attacked and became entangled in the feather; the feather was immediately sealed in a zip lock bag with breathing holes and placed in the freezer at $-10{ }^{\circ} \mathrm{C}$ for approximately $5 \mathrm{~min}$ to coldanaesthetise the bees.

Once immobilised, the anaesthetised bees were transferred to the experimental setup for analysing aggressive behaviour. On average, it took bees 34 min to wake up inside the setup. The experiment was only started once the bees were fully awake and moved around normally. If a bee took longer than $5 \mathrm{~min}$ to recover from the cold treatment, the experiment was cancelled. After an experiment concluded, all bees were released close to their hive entrance.

\subsection{Experimental setup}

A behavioural assay was developed to investigate aggressive behaviour in individual honeybees under controlled laboratory conditions. The experimental setup consisted of a large Petri dish (150 mm ID) with moist filter paper at the bottom and fly mesh on top to enclose the dish. The dish was placed on supports creating a $3 \mathrm{~cm}$ high space underneath the dish (Figure 1). A dummy made of a 2-cm-long stirring magnet wrapped in a black cloth held in place by strips of yellow tape was placed inside the dish. A controlling magnet in the space underneath the dish was used to move the dummy within a $4 \mathrm{~cm}$ circle inside the setup. Depending on the experiment, the dummy was untreated or treated with $5 \mu \mathrm{L}$ IAA (Sigma Aldrich). For each test, a new, clean Petri dish was used with fresh filter paper and a new dummy to prevent potential contamination from previous tests.

Honeybees collected as described above were placed in the setup two at a time. The bees were analysed in pairs to observe social interactions as a measure for normal behaviour, as well as aggressive behaviours towards the dummy. Using a video camera, the setup was recorded from above from the moment both bees were fully recovered from cold treatment. Recordings lasted either 5 or $2 \mathrm{~min}$ depending on the specific experiment (see below 2.3 to 2.5 and Table II). The video recordings were subsequently analysed continuously as follows: Behaviours ranging from non-aggressive (e.g. grooming, eating, walking) to increasingly aggressive (e.g. pacing, raising abdomen, buzzing, biting, stinging; Free 1987) were defined and assigned an 


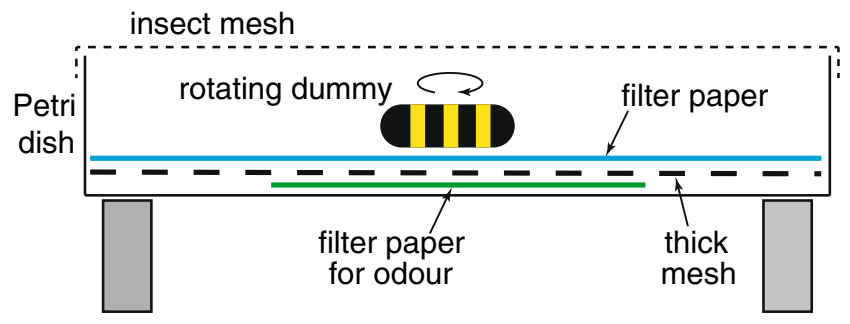

Figure 1. Schematic of experimental setup to investigate aggressive behaviour in honeybees under controlled laboratory conditions (Petri dish aggression assay). Side view of $15 \mathrm{~cm}$ Petri dish on supports allowing a $3 \mathrm{~cm}$ space underneath the dish to move the controlling magnet for the moving dummy, which was made of black cloth wrapped around a $2 \mathrm{~cm}$ long stirring magnet held in place by strips of yellow tape. A filter paper (blue) on the bottom of the dish was moistened with water to keep the atmosphere inside the dish humid. A second, smaller filter paper (green) was used to apply plant odours; it was separated by thick mesh to prevent direct contact with the top filter paper and the walking bees.

aggression value, with 0 being a non-aggressive behaviour, and 6 being the most aggressive behaviours such as stinging and biting (Table I). Aggression values were chosen based on the behavioural stages described for aggression and colony defense in honeybees (Collins et al. 1980).

For analysis, the total number of seconds that a bee displayed each behaviour during the entire recording was determined, and these numbers were then multi-

Table I. Honeybee behaviours and their aggression values used for determining aggressiveness in a Petri dish assay.

\begin{tabular}{ll}
\hline Behaviour & Aggression value \\
\hline Walking & 0 \\
Grooming (self, other) & 0 \\
Eating & 0 \\
Sitting still $^{\mathrm{a}}$ & 1 \\
Raising abdomen/high stance & 1 \\
Pacing & 2 \\
Buzzing & 3 \\
Flying & 4 \\
Contacting dummy & 5 \\
Stinging/biting dummy & 6 \\
\hline
\end{tabular}

${ }^{\text {a }}$ Sitting still can be a sign of alertness as part of defensive behaviour plied by the assigned aggression value for the respective behaviours (Table I). The results were added up and then divided by the number of minutes an assay lasted, resulting in a final aggression score. This method ensured all aggression scores could be directly compared between experiments. To illustrate a theoretical example: A bee that spent the entire test stinging the dummy would have an aggression score of 360 , irrespective of whether the experiment lasted $5 \mathrm{~min}$ (300 s multiplied by an aggression value of ' 6 ' for stinging and divided by 5) or $2 \mathrm{~min}$ ( $120 \mathrm{~s}$ multiplied by ' 6 ' and divided by 2). If a bee spent more time with aggressive behaviours than with, for instance, walking or grooming, its aggression score would be higher.

\subsection{Aggression assay}

To determine the optimal combination of olfactory, visual and mechanical stimuli required to elicit aggression in the Petri dish assay described above, we conducted three tests: test 1 , with the dummy present but not moving; test 2, with the dummy moving continuously in the Petri dish; and test 3, with the dummy moving and $5 \mu \mathrm{L}$ of the honeybee alarm pheromone IAA (Sigma-Aldrich) applied on the dummy. Each test was repeated nine times with a new pair of bees, that is, 18 individual bees in total were used per test (Table II). An aggression score was calculated for each and every individual bee of a pair, and scores for all 18 bees of a test were pooled for analysis. 
Table II. Overview of experimental conditions and odour treatments investigating honeybee aggressive behaviour in a Petri dish assay.

\begin{tabular}{|c|c|c|c|c|c|c|}
\hline Experiment & $\begin{array}{l}\text { Odour in } \\
\text { cage }\end{array}$ & $\begin{array}{l}\text { Dummy } \\
\text { moving }\end{array}$ & $\begin{array}{l}\text { Odour on } \\
\text { dummy }\end{array}$ & $\begin{array}{l}\text { Odour on filter } \\
\text { paper }\end{array}$ & $\begin{array}{l}\text { Test } \\
{[\mathrm{min}]}\end{array}$ & $\begin{array}{l}\text { No. } \\
\text { bees }\end{array}$ \\
\hline \multicolumn{7}{|c|}{ Aggression assay } \\
\hline Test 1 & $\mathrm{n} / \mathrm{a}$ & No & - & - & 5 & 18 \\
\hline Test 2 & $\mathrm{n} / \mathrm{a}$ & Yes & - & - & 5 & 18 \\
\hline Test 3 & $\mathrm{n} / \mathrm{a}$ & Yes & IAA & - & 5 & 18 \\
\hline \multicolumn{7}{|c|}{ Short-term exposure to lavender } \\
\hline Test 1 & $\mathrm{n} / \mathrm{a}$ & Yes & IAA & - & 5 & 12 \\
\hline Test 2 & $\mathrm{n} / \mathrm{a}$ & Yes & IAA & Lavender & 5 & 12 \\
\hline Test 3 & $\mathrm{n} / \mathrm{a}$ & Yes & - & Lavender & 5 & 12 \\
\hline \multicolumn{7}{|c|}{ Short-term exposure to Praescent } \\
\hline Test 1 & $\mathrm{n} / \mathrm{a}$ & Yes & IAA & - & 5 & 12 \\
\hline Test 2 & $\mathrm{n} / \mathrm{a}$ & Yes & IAA & Praescent & 5 & 12 \\
\hline Test 3 & $\mathrm{n} / \mathrm{a}$ & Yes & - & Praescent & 5 & 12 \\
\hline \multicolumn{7}{|c|}{ Short-term exposure (solvent control) } \\
\hline Test 1 & $\mathrm{n} / \mathrm{a}$ & Yes & IAA & - & 5 & 10 \\
\hline Test 2 & $\mathrm{n} / \mathrm{a}$ & Yes & IAA & TEC & 5 & 10 \\
\hline Test 3 & $\mathrm{n} / \mathrm{a}$ & Yes & - & TEC & 5 & 10 \\
\hline \multicolumn{7}{|c|}{ Short-term exposure (IAA control) } \\
\hline Test 1 & $\mathrm{n} / \mathrm{a}$ & Yes & IAA & - & 5 & 10 \\
\hline Test 2 & $\mathrm{n} / \mathrm{a}$ & Yes & IAA & - & 5 & 10 \\
\hline Test 3 & $\mathrm{n} / \mathrm{a}$ & Yes & IAA & - & 5 & 10 \\
\hline \multicolumn{7}{|c|}{ Long-term exposure to odours } \\
\hline Test 1 & Water & Yes & IAA & - & 2 & 33 \\
\hline Test 2 & TEC & Yes & IAA & - & 2 & 27 \\
\hline Test 3 & IAA & Yes & IAA & - & 2 & 29 \\
\hline Test 4 & lavender & Yes & IAA & - & 2 & 31 \\
\hline Test 5 & IAA + lavender & Yes & IAA & - & 2 & 28 \\
\hline Test 6 & Praescent & Yes & IAA & - & 2 & 28 \\
\hline Test 7 & IAA+Praescent & Yes & IAA & - & 2 & 27 \\
\hline
\end{tabular}

$I A A$ isoamyl-acetate, TEC triethyl citrate, the solvent for lavender and Praescent

\subsection{Short-term exposure to plant odours}

The same experimental setup was used, but an additional small filter paper $(9 \mathrm{~cm}$ diameter) was placed in the bottom of the dish underneath the standard filter paper. It was separated by a thick mesh from the larger filter paper on top preventing the two filter papers touching each other (Figure 1). To test for the aggression-modulating effect of plant-derived odours, this additional filter paper was treated with $30 \mu \mathrm{l}$ of either lavender $(0.03 \%$ lavender essential oil from Oil Garden Essential Oils, in triethyl citrate from Sigma Aldrich) or Praescent ${ }^{\mathrm{TM}}$ (combination of $0.03 \%$ cis-3hexanol, $0.03 \%$ trans-2-hexenal, $0.015 \%$ alpha-pinene in triethyl citrate, all from Sigma-Aldrich). Due to their volatility, the plant odours filled the Petri dish odour space, but the thick mesh between the filter paper with plant odour and the filter paper on which the bees 
walked prevented bees from coming into direct contact with the odours during walking. For both lavender and Praescent, a series of three consecutive tests each lasting 5 min were conducted using the following odour treatments (Table II): (1) IAA on the dummy, but no odour applied on the filter paper; (2) IAA on the dummy and $30 \mu \mathrm{L}$ plant odour on the filter paper; (3) no IAA on the dummy but $30 \mu \mathrm{L}$ plant odour on the filter paper. The first test established the general aggression score; the second test determined the effect of the plant odour on aggression, and the third test determined the effect of the plant odour alone. All three tests of a series were run consecutively using the same two bees. Only bees that reacted aggressively in the first test, i.e. showed pacing, flying, dummy contact and/or stinging behaviours for the majority of the $5 \mathrm{~min}$, were used for the next two tests of a series. Each test of a series was conducted in a clean Petri dish with all experimental materials replaced. The bees were anaesthetised for $10 \mathrm{~min}$ at $4{ }^{\circ} \mathrm{C}$ in between tests in order to transfer them into the dish for the next test. Each series was repeated six times with a new pair of bees, until a total of 12 bees were tested for both the lavender and the Praescent series. An equivalent series of three consecutive tests was conducted using $30 \mu \mathrm{L}$ triethyl citrate (TEC) to exclude possible effects of the solvent. For this solvent control, ten bees in total were tested (Table II). To investigate whether the repeated anaesthetisation of the bees between tests affected aggressiveness, a further control experiment was conducted with three consecutive 5-min tests, each of which had IAA applied on the dummy, but no plant odour on the filter paper. A total of ten bees were tested for the IAA-control series (Table II).

\subsection{Long-term exposure to plant odours}

Guard bees caught as described above were placed in groups of 10-12 individuals in cages $(\mathrm{L} \times \mathrm{H} \times \mathrm{W}$, $15 \times 10 \times 3 \mathrm{~cm}$ ) with diluted honey ad lib as food source. The honey was provided via a $15 \mathrm{~mL}$ falcon tube inserted into the cage. The tube had a large hole on the side through which the bees could access the honey. A second $15 \mathrm{~mL}$ falcon tube inserted into the cage contained a cotton wool ball with odours, as specified below, which evaporated through 12 ventilation holes in the tube. Seven cages were used differing by type of odour on the cotton ball: (1) $150 \mu \mathrm{L}$ of $\mathrm{H}_{2} \mathrm{O}$ (control), (2) $150 \mu \mathrm{L}$ of triethyl citrate (solvent control), (3) $30 \mu \mathrm{L}$ of IAA, (4) $150 \mu \mathrm{L}$ of lavender, (5) $30 \mu \mathrm{L}$ of IAA plus $150 \mu \mathrm{L}$ of lavender, (6) $150 \mu \mathrm{L}$ of Praescent and (7) $30 \mu \mathrm{L}$ of IAA plus $150 \mu \mathrm{L}$ of Praescent (Table II). Each cage was fitted into a dark box that was taped up to ensure the odours were restricted to the specific cage. Cages in their boxes were placed for $48 \mathrm{~h}$ into an incubator at $34{ }^{\circ} \mathrm{C}$ and $70 \%$ R.H. Both the food and the odour tubes were replaced every $12 \mathrm{~h}$. After $48 \mathrm{~h}$, bees were tested in pairs for aggressiveness in the Petri dish assay: $5 \mu \mathrm{L}$ IAA was applied on a moving dummy, and bee behaviour was recorded for $2 \mathrm{~min}$ to investigate whether long-term exposure to IAA or the plant odours had affected their aggression levels (Table II). The experiment was repeated three times until approximately 30 bees were tested for each odour treatment.

\subsection{Statistics}

Aggression scores for all bees from an experimental treatment were pooled, and the mean and standard deviation were calculated. For the aggression assay and experiments with short-term exposure to plant odours, we used independent two sample $t$ test (separate groups of bees) and paired $t$ test (same group of bees tested consecutively), respectively, as well as one-way ANOVA to analyse differences in aggression scores between treatments. For experiments with long-term exposure to plant odours, aggression scores were analysed using one-way ANOVA with odour exposure as factor, followed by post hoc paired $t$ test comparing each exposure group to the water (control) group. For multiple comparisons, Bonferroni correction was applied to adjust the $\alpha$-level in order to avoid Type I errors.

\section{RESULTS}

\subsection{Aggression assay}

The aggression scores for the three tests showed significant differences between assay conditions depending on the sensory stimuli involved. There was no clear aggression observed and no statistical difference in aggression scores between the two odour-less control tests 1 (dummy not moving) and 2 (dummy moving), 
albeit the aggression score was slightly higher when the dummy was moving (Figure 2). When IAA was added onto the moving dummy (test 3), it elicited significant aggressive behaviour (Figure 2). These conditions, namely a moving dummy with IAA applied, were used for the subsequent tests investigating the effect of plant odours on aggressive behaviour in honeybees.

\subsection{Short-term exposure to plant odours}

Using the same pair of bees over three consecutive tests (Table II) allowed direct comparisons of the effects of short-term exposure to plant odours on aggressive behaviour in the same individuals. When no plant odour was present, the aggression scores were high in both the lavender and the Praescent test series, with the IAA-treated moving dummy being

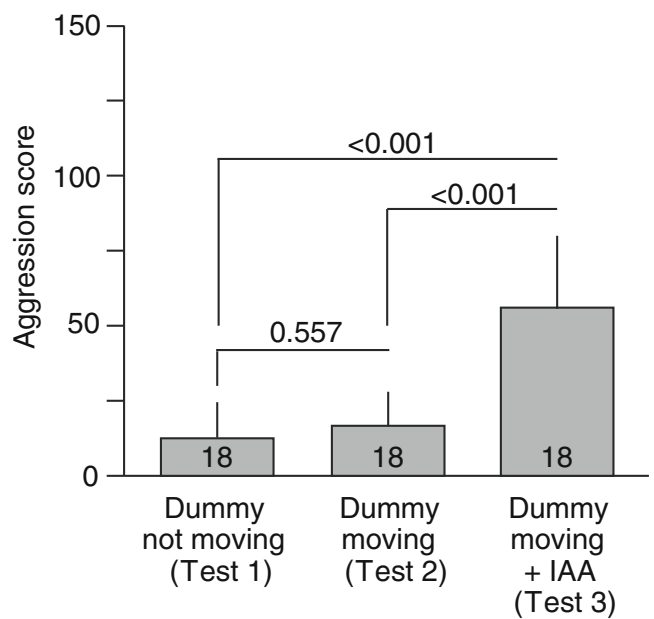

Figure 2. Honeybee aggression score $(m e a n \pm S D)$ determined in a Petri dish aggression assay investigating the role of visual, mechanical and olfactory triggers for aggression. Tests 1 and 2 included odourless conditions with either a stationary (test 1 ) or moving (test 2) dummy. Test 3 used $5 \mu \mathrm{L}$ of the honeybee alarm pheromone isoamyl-acetate (IAA) on a moving dummy. For further details, see Table II. Numbers inside bars are the total number of tested honeybees per group; numbers on top of lines are $p$ values (independent two sample $t$ test with Bonferroni correction, $\alpha=0.017$ ). contacted frequently (Figure 3a). When lavender was added to the Petri dish, the aggression score dropped significantly, that is, aggressive behaviour was reduced in presence of lavender odour. The same effect was observed when Praescent was added to the Petri dish, with the aggression-reducing effect of Praescent slightly stronger than for lavender $(P=0.046$, independent two-sample $t$ test). When only lavender or Praescent were present in the Petri dish, but no IAA applied on the dummy, the bees had very low aggression scores (Figure 3a), comparable to those of tests 1 and 2 of the first experiment (Figure 2). Also, bees did not display any unusual behaviour when exposed to these two plant odours, but walked around and groomed each other normally.

The test series using TEC (solvent control) showed no reduction in aggression when TEC was added (Figure 3b), confirming that the effect observed for lavender and Praescent was not due to the solvent. TEC alone without IAA led to a low aggression score as expected when IAA is not present (see also Figure 2, Test 2) and had no effect on the bees' general behaviour. The final series with IAA on the dummy in each test, but no plant odours present (IAA control) resulted in consistently high aggression scores in all three tests with no statistical difference between the tests (Figure 3b). These scores were in the same range and statistically not different from those of the first test of the lavender series and Praescent series when only IAA was present $\left(F_{(4,49)}=0.99, P=0.417\right.$, ANOVA). That is, the repeated use of the same two bees in three consecutive tests did not affect aggressiveness. This result also counteracts the potential issue that the three tests of a series were always conducted in the same sequence and not randomised. Aggressiveness being equal in all three IAA-tests of the final series ruled out that test sequence could have an effect.

\subsection{Long-term exposure to plant odours}

ANOVA revealed a significant difference between the treatment groups depending on 

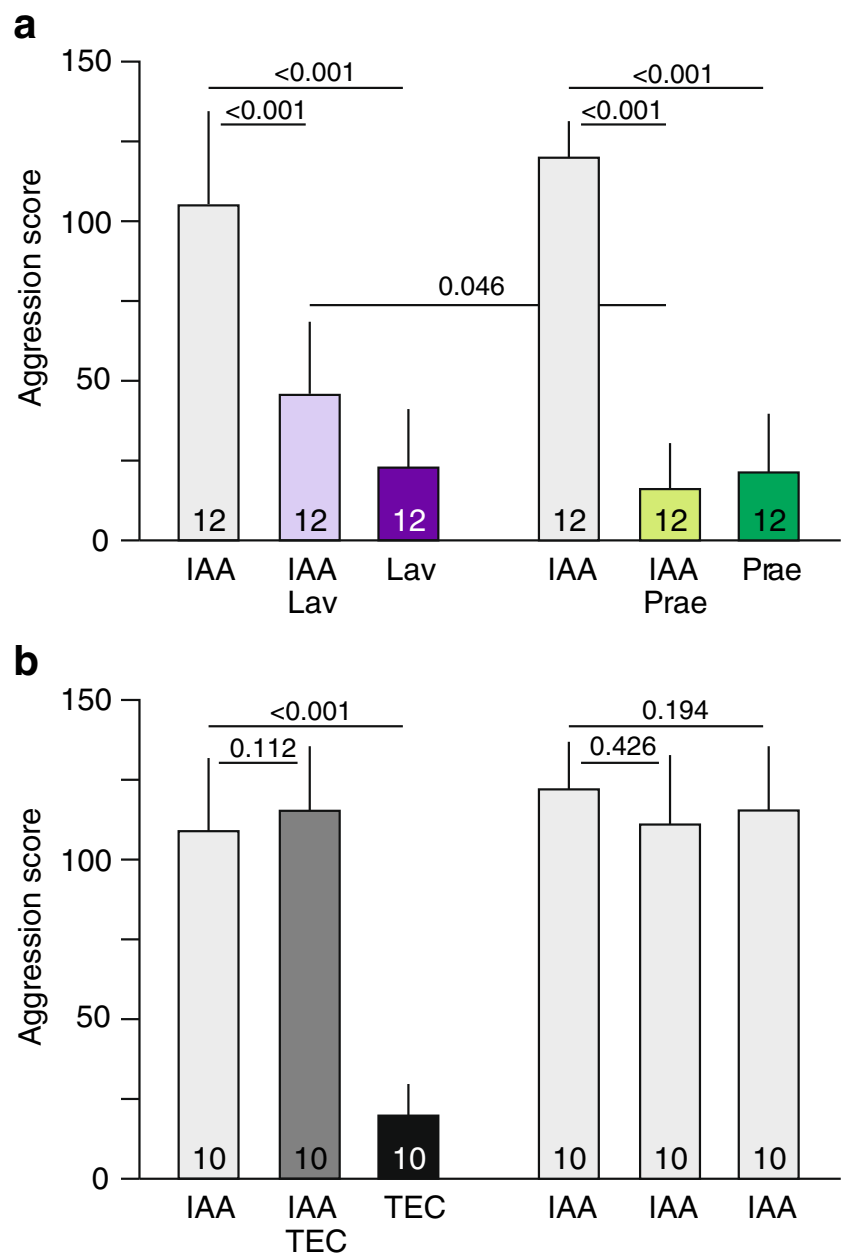

Figure 3. Honeybee aggression score (mean $\pm \mathrm{SD}$ ) during short-term exposure to plant odours. a Exposure to lavender (Lav) and Praescent (Prae) with and without the alarm pheromone isoamyl-acetate (IAA); b exposure to the solvent triethyl citrate (TEC) with and without isoamyl-acetate, and to isoamyl-acetate on its own (IAA). The three bars of a group represent three consecutive 5-min tests with the same pair of bees. For further details regarding the odour treatments, see text and Table II. Numbers inside bars are the total number of tested honeybees per group; numbers on top of lines are $p$ values (paired $t$ test with Bonferroni correction $(\alpha=0.025)$ for comparing three tests within a group and independent two-sample $t$ test $(\alpha=0.05)$ for comparing the second test between the lavender and Praescent groups).

the type of odour they were exposed to $\left(F_{(6,197)}=28.32, P<0.001\right)$. When bees were exposed for $48 \mathrm{~h}$ to water, the solvent TEC or IAA, they showed high aggression scores (Figure 4), comparable to the aggression scores found in the other experiments. The IAAexposed group had a slightly lower aggression score than the water-exposed and TEC-exposed groups, but the difference was statistically not significant. However, all four groups of bees that were exposed for $48 \mathrm{~h}$ to lavender or Praescent, with or without IAA, showed significantly reduced aggressiveness compared with the controls (water and TEC) (Figure 4). 


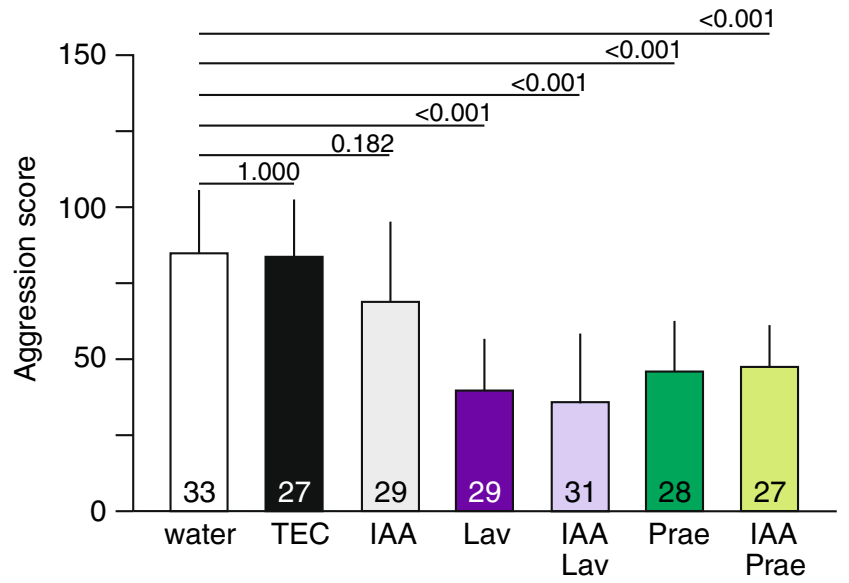

Figure 4. Honeybee aggression score (mean $\pm \mathrm{SD}$ ) after 48-h exposure to water (control), triethyl citrate (TEC, solvent control), honeybee alarm pheromone isoamyl-acetate (IAA), the plant odours lavender (Lav) or Praescent (Prae) or combinations of pheromone and plant odours. Bees were tested in a 2-min aggression assay with IAA applied on a moving dummy. Further details, see text and Table II. Numbers inside bars are the total number of tested honeybees per group; numbers on top of lines are $p$ values (post hoc paired $t$ test with Bonferroni correction, $\alpha=0.008$ ).

There was no difference in survival rates between treatment groups, with $87-92 \%$ of bees surviving $48 \mathrm{~h}$ in the cage irrespective of odour treatment.

\section{DISCUSSION}

\subsection{Development of a novel aggression assay}

We developed a novel assay to evaluate honeybee aggression under controlled conditions in a Petri dish. The data from our first experiment showed that the mere presence of an object even if moving is not sufficient to elicit full aggression in honeybees in this assay, supporting earlier observations that identification and attack of a target relies on a complex set of stimuli (Free 1987; Schmidt 1998). In our assay, the lack of aggressive response towards the dummy alone might have been due to the target being comparatively small and its movements too slow not necessarily touching the bees directly. Indeed, new experiments from our laboratory recently showed that using a modified leather-covered dummy with feathery extensions that is moved by an automated, vibrating stepping-motor and frequently touches the bees in the dish elicits a significantly stronger aggressive response (unpublished data). To ensure that sufficient aggression is reliably triggered throughout tests, it would be preferable to use this modified dummy for future studies.

The addition of the alarm pheromone component IAA to the dummy triggered the full behavioural sequence and led to stinging of the object, demonstrating the importance of olfactory cues in eliciting honeybee aggressive behaviour and confirming its well-established effect on honeybee aggression (Ghent and Norman 1962; Free 1987; Schmidt 1998; Balderrama et al. 2002). In our study, we used $5 \mu \mathrm{L}$ of pure IAA, which was the minimal concentration required to trigger full aggression in our setup. However, in a setup using a different "stronger" dummy, such as the one mentioned above, it is likely that much less IAA is required. The aggression score elicited by IAA in the first experiment (Figure 2) was slightly lower than the aggression scores triggered by IAA in the other two experiments (Figures 3 and 4). This is possibly due to the fact that in the 
first experiment we used random bees, while in the other experiments we tested guard bees only, which are the ones initiating aggressive behaviour and colony defense (Hunt 2007). Inter-individual variability with respect to aggressiveness threshold is a problem for all aggression assays. Using the SER assay, a comparative study showed that individuals within a honeybee colony differ in their responsiveness thresholds to electric shock, with guard bees having a higher threshold, i.e. being less responsive (Roussel et al. 2009). Our assay did not use electric shock as stimulus, and we cannot conclude with certainty that guard bees also have a higher response threshold to the moving dummy, as sensitivities to sensory stimuli from different modalities and/or of different hedonic value are not necessarily correlated (Roussel et al. 2009; Tedjakumala and Giurfa 2013). The fact that the aggression scores in the first experiment with random bees were slightly lower than those in the other experiments with guard bees suggests that the guards may actually be more responsive in case of the dummy stimulus. This aspect could be investigated by directly comparing guards and foragers in our assay. The choice of test individuals, however, depends on the research question. If one were to assess an entire hive's aggressiveness, bees from all castes should be tested in numbers of at least 30 per caste. In contrast, if the mechanisms underlying regulation of aggressive behaviour are to be investigated, one should attempt to assay a group of more or less homogeneous individuals, for example, by using only guard bees as done here, or by using age-marked bees.

Our assay records all behavioural stages of aggression, providing a more detailed measure for the level of aggressiveness than merely counting stings. Scoring several behaviours on a relative scale from 1 to 6 may seem rather subjective and prone to variability. However, it has been shown that aggression assays using behavioural rating systems are more reliable and much less variable in their results than generic stinging assays (Guzman-Novoa et al. 2003). The one disadvantage of our new assay is the fact that video analysis of multiple behaviours is rather time-consuming. Nonetheless, our Petri dish assay could be useful to directly compare aggressiveness between different bee castes, hives, races or species, or determine the effect of environmental conditions and genetic factors on aggressiveness in a controlled setup. This assay also allows investigation into the functional role of aggression triggers such as pheromones, or into the effects of aggressionmodulating treatments including plant odours, which was the second aim of our study.

\subsection{Short-term exposure to plant odours}

When plant odours were added to the Petri dish, they significantly reduced aggression towards the moving target treated with IAA. This effect was recorded for both lavender and Praescent. Praescent had a slightly stronger attenuating effect on aggression; however, given the variability of behavioural data, this might merely be due to the comparatively small number of bees tested. Clearly, both plant odours significantly decrease aggressive behaviour during short-term exposure. This provides scientific support to anecdotal reports that lavender oil applied onto hands or gloves is an effective way of reducing stinging during beekeeping routines, suggesting Praescent as suitable alternative. It also shows, for the first time, that Praescent not only attenuates stress in vertebrates (Akutsu et al. 2002, 2003; Aou et al. 2005; Kim et al. 2005; Tokumo et al. 2006; Fukada et al. 2007; Fujita et al. 2010), but also has a modulating effect on insect aggression.

One could argue that mixing IAA and plant odours in this assay may have simply "overwhelmed" the bee's olfactory system, such that it no longer was able to detect IAA. This is an unlikely scenario, considering the small volume of plant odour used $(30 \mu \mathrm{l})$ within the comparatively large odour space of the Petri dish and the fact that even the human experimenter could still detect both IAA and the plant odours. But clearly, a detailed study investigating different ratios of IAA and plant odours is needed to determine a dose-response curve for lavender and Praescent in context of aggressive behaviour. One may further argue that the 
reduced aggression was due to the bees being repeatedly cooled and anesthetised or that they simply got used to the setup during repeated testing over three trials. However, the control experiment of three consecutive IAA-tests without plant odours clearly shows that the testing procedure did not reduce aggression over three tests. Also, the presence of plant odours did not compromise the general behaviour of bees, as demonstrated through the third tests using only plant odours. Our results thus clearly demonstrate that lavender and Praescent attenuate aggression in bees. However, the mechanisms of how these plant odours interact with or counteract IAA, and how this results in reduced aggressiveness remains to be investigated.

\subsection{Long-term exposure to plant odours}

Long-term exposure over $48 \mathrm{~h}$ to both lavender and Praescent also had an attenuating effect on aggressive behaviour in honeybees. Bees kept with water, the solvent TEC or IAA for $48 \mathrm{~h}$ showed high aggression scores comparable to those of the other experiments. That is, the 48-h cage treatment did not affect aggressive behaviour in a significant way. Interestingly, bees kept with IAA in the cage had a slightly lower aggression score than the control bees kept with water or with the solvent. This is consistent with earlier reports that continuous exposure to IAA can reduce aggression (Al-Sa'ad et al. 1985; Free 1988) and change the responsiveness to nociceptive stimuli such as electric shocks (Núñez et al. 1998; Balderrama et al. 2002). It has been suggested that IAA exposure potentially activates an opioid system in honeybees inducing an analgesia-like state, thus enhancing tolerance to noxious stimuli and decreasing responsiveness to electric shocks (Núñez et al. 1998; Balderrama et al. 2002). This could explain the slightly lowered aggressiveness in our movingdummy-assay after $48 \mathrm{~h}$ exposure to IAA.

Notably, all groups that were exposed to lavender or Praescent for $48 \mathrm{~h}$, with or without IAA, showed less aggression compared with the control with water. It is important to note that the aggression assay conducted after long-term exposure did not contain any plant odours in the Petri dish, but only IAA on the moving dummy. That is, the attenuating effect on bee aggression was entirely due to the $48 \mathrm{~h}$ exposure to the plant odours while kept in the cage. This also rules out, at least partially, the argument raised above for the short-term assay that mixing of IAA and plant odours could possibly mask IAA and thus be the reason for reduced aggression. In the long-term exposure experiment, no mixing occurred, as both kinds of odours are not coincident temporally, but aggression was still reduced. Clearly, extended exposure to plant odours seems to change how bees respond to alarm triggers, such as moving objects and IAA. Whether long-term exposure to plant odours induces a similar analgesia-like state in bees as does exposure to IAA (Núñez et al. 1998) remains to be investigated.

\section{CONCLUSION}

Using a novel assay to investigate aggressiveness in honeybees under controlled laboratory conditions, we showed that certain plant odours attenuate aggression both during shortterm and long-term exposure. Our study raised a number of new questions, the most intriguing being the molecular and physiological mechanisms underlying the effect of plant odours on honeybee aggression, and whether different mechanisms are at play during short-term and long-term exposure. This question is topic of future investigations, together with the question whether the effect is unique to lavender and Praescent, or generic for plant odours. Although our study is only a first step into unravelling the complex olfactory mechanisms regulating honeybee aggression, it suggests an intriguing yet simple way to modulate aggressive behaviour in honeybees, namely the use of environmentally derived plant odours. These could be either applied on a person when handling bees or aggressive hives could be treated with plant odours. 


\section{ACKNOWLEDGMENTS}

We thank Naila Even for technical advice on catching guard bees and Emma Condon for help with preliminary experiments. We are grateful to Morgane Nouvian and two anonymous reviewers for providing critical feedback on an earlier version of the manuscript. This work was supported by the Queensland Brain Institute, The University of Queensland. All experiments were performed according to the standards for animal experimentation in Australia.

Un nouvel essai pour évaluer la modulation olfactive du comportement agressif de l'abeille

Apis mellifera / agression / acétate d'isoamyle / olfaction / essai en laboratoire / odeur de plantes

Ein neuer Versuchsansatz zur Untersuchung von olfaktorischer Modulierung aggressiven Verhaltens bei der Honigbiene

\section{Aggression / Honigbiene / IAA / Olfaktorik / Verhalten / Pflanzendüfte}

\section{REFERENCES}

Al-Sa'ad, B.N., Free, J.B., Howse, P.E. (1985) Adaptation of worker honeybees (Apis mellifera) to their alarm pheromone. Physiol. Entomol. 10, 1-14

Akutsu, H., Kikusui, T., Takeuchi, Y., Sano, K., Hatanaka, A., Mori, Y. (2002) Alleviating effects of plant-derived fragrances on stress-induced hyperthermia in rats. Physiol. Behav. 75, 355-360

Akutsu, H., Kikusui, T., Takeuchi, Y., Mori, Y. (2003) Effects of alpha-pinene odor in different concentrations on stress-induced hyperthermia in rats. J. Vet. Med. Sci. 65, 1023-1025

Aou, S., Mizuno, M., Matsunaga, Y., Kubo, K., Li, X.L., Hatanaka, A. (2005) Green odor reduces pain sensation and fatigue-like responses without affecting sensorimotor function. Chem. Senses 30, 262-263

Arechavaleta-Velasco, M.E., Hunt, G.J. (2003) Genotypic variation in the expression of guarding behavior and the role of guards in the defensive response of honey bee colonies. Apidologie 34, 439-447

Balderrama, N., Nunez, J., Guerrieri, F., Giurfa, M. (2002) Different functions of two alarm substances in the honeybee. J. Comp. Physiol. A 188, 485-491

Breed, M.D., Robinson, G.E., Page, R.E. (1990) Division of labor during honey bee colony defense. Behav. Ecol. Sociobiol. 27, 395-401
Breed, M.D., Guzman-Novoa, E., Hunt, G.J. (2004) Defensive behavior of honey bees: organization, genetics, and comparisons with other bees. Annu. Rev. Entomol. 49, 271-298

Collins, A.M., Rinderer, T.E., Tucker, K.W., Sylvester, H.A., Lacket, J.J. (1980) A model of honey bee defensive behaviour. J. Apic. Res. 19, 383-387

Collins, A.M., Rinderer, T.E., Harbo, J.R., Bolten, A.B. (1982) Colony defense by africanized and European honey bees. Science 218, 72-74

Even, N., Devaud, J.M., Barron, A.B. (2012) General stress responses in the honey bee. Insects 3, 1271-1298

Francoy, T.M., Wittmann, D., Steinhage, V., Drauschke, M., Muller, S., et al. (2009) Morphometric and genetic changes in a population of Apis mellifera after 34 years of Africanization. Genet. Mol. Res. 8, 709-717

Free, J.B. (1961) The stimuli releasing the stinging behaviour of honeybees. Anim. Behav. 9, 193-196

Free, J.B. (1987) Pheromones of social bees. Cornell University Press, Ithaca

Free, J.B. (1988) Adapting honeybees (Apis mellifera L.) to synthetic alarm pheromones to reduce aggression. J. Apic. Res. 27, 227-229

Fujita, S., Ueki, S., Miyoshi, M., Watanabe, T. (2010) "Green odor" inhalation by stressed rat dams reduces behavioral and neuroendocrine signs of prenatal stress in the offspring. Horm. Behav. 58, 264-272

Fukada, M., Kaidoh, T., Ito, A., Yano, T., Hayashibara, C., Watanabe, T. (2007) "Green odor" inhalation reduces the skin-barrier disruption induced by chronic restraint stress in rats: physiological and histological examinations. Chem. Senses 32, 633-639

Ghent, R.L., Norman, E.G. (1962) A chemical alarm releaser in honey bee stings (Apis mellifera L.). Psyche 69, 1-6

Guzman-Novoa, E., Page, R.E., Spangler, H.G., Erickson, E.H. (1999) A comparison of two assays to test the defensive behaviour of honey bees (Apis mellifera). J. Apic. Res. 38, 205-209

Guzman-Novoa, E., Prieto-Merlos, D., Uribe-Rubio, J.L., Hunt, G.J. (2003) Relative reliability of four field assays to test defensive behaviour of honey bees (Apis mellifera). J. Apic Res. 42, 42-46

Guzman-Novoa, E., Hunt, G.J., Uribe-Rubio, J.L., Prieto-Merlos, D. (2004) Genotypic effects of honey bee (Apis mellifera) defensive behavior at the individual and colony levels: the relationship of guarding, pursuing and stinging. Apidologie 35, 1534

Hunt, G.J. (2007) Flight and fight: a comparative view of the neurophysiology and genetics of honey bee defensive behavior. J. Insect Physiol. 53, 399-410

Kim, J., Ishibshi, M., Nakajima, K., Aou, S., Hatanaka, A., Oomura, Y., Sasaki, K. (2005) Effects of green odor on expression of Fos-immunoreactivity in the 
paraventricular nucleus of the thalamus in forced swimming rats. Chem. Senses 30, 266-267

Kolmes, S.A., Fergusson-Kolmes, L. (1989) Stinging behavior and residual value of worker honey bees (Apis mellifera). J. NY Entomol. Soc. 97, 218-231

Lenoir, J.C., Laloi, D., Dechaume-Moncharmont, F.X., Solignac, M., Pham, M.H. (2006) Intracolonial variation of the sting extension response in the honeybee Apis mellifera. Insectes Soc. 53, 80-85

Moore, A.J., Breed, M.D., Moor, M.J. (1987) The guard honey-bee: ontogeny and behavioral variability of workers performing a specialized task. Anim. Behav. 35, 1159-1167

Núñez, J.A., Almeida, L., Balderrama, N., Giurfa, M. (1998) Alarm pheromone induces stress analgesia via an opioid system in the honeybee. Physiol. Behav. 63, 75-80

Paxton, R.J., Sakamoto, C.H., Rugiga, F.C.N. (1994) Modification of honey-bee (Apis mellifera $\mathrm{L}$.) stinging behavior by within-colony environment and age. J. Apic. Res. 33, 75-82

Roussel, E., Carcaud, J., Sandoz, J.C., Giurfa, M. (2009) Reappraising social insect behavior through aversive responsiveness and learning. Plos One 4, e4197

Schmidt, J.O. (1998) Mass action in honey bees: alarm, swarming and the role of releaser pheromones. In: VanderMeer, R.K., Breed, M.D., Espelie, K.E., Winston, M.L. (eds.) Pheromone communication in social insects, pp. 257-290. Westview Press, Boulder

Southwick, E.E., Moritz, R.F.A. (1985) Metabolic response to alarm pheromone in honey bees. J. Insect Physiol. 31, 389-392
Southwick, E.E., Moritz, R.F.A. (1987) Effects of meteorological factors on defensive behaviour of honey bees. Int. J. Biometeorol. 31, 259-265

Tedjakumala, S.R., Giurfa, M. (2013) Rules and mechanisms of punishment learning in honey bees: the aversive conditioning of the sting extension response. J. Exp. Biol. 216, 2985-97

Tokumo, K., Tamura, N., Hirai, T., Nishio, H. (2006) Effects of (Z)-3-hexenol, a major component of green odor, on anxiety-related behavior of the mouse in an elevated plus-maze test and biogenic amines and their metabolites in the brain. Behav. Brain Res. 166, 247-252

Uribe-Rubio, J.L., Guzman-Novoa, E., Vazquez-Pelaez, C.G., Hunt, G.J. (2008) Genotype, task specialization, and nest environment influence the stinging response thresholds of individual Africanized and European honeybees to electrical stimulation. Behav. Genet. 38, 93-100

Visser, J.H., van Straten, S., Maarse, H. (1979) Isolation and identification of volatiles in the foliage of potato, Solanum tuberosum, a host plan of the Colorado beetle, Leptinotarsa decemlienata. J. Chem. Ecol. 5, 13-25

Visscher, P.K., Vetter, R.S., Robinson, G.E. (1995) Alarm pheromone perception in honey bees is decreased by smoke (Hymenoptera: Apidae). J. Insect Behav. 8, 11-18

Wager, B.R., Breed, M.D. (2000) Does honey bee sting alarm pheromone give orientation information to defensive bees? Ann. Ent. Soc. Am. 93, 1329-1332

Winston, M.L. (1991) The biology of the honey bee. Harvard University Press, Cambridge 